\title{
Evidence for Point Transformations in Photoactive Molecular Crystals by the Photoinduced Creation of Diffuse Diffraction Patterns
}

\author{
Jörg Hallmann, ${ }^{\dagger}$ Rene More, ${ }^{\dagger}$ Wolfgang Morgenroth, ${ }^{\ddagger, \S}$ Carsten Paulmann, ${ }^{\dagger, \perp}$ Qingyu Kong, ${ }^{\text {II }}$ \\ Michael Wulff, ${ }^{\text {II }}$ and Simone Techert*, \\ ${ }^{\dagger}$ IFG Structural Dynamics of (Bio)chemical Systems, Max Planck Institute for Biophysical Chemistry, 37070 Göttingen, Germany \\ *c/o HASYLAB at DESY, Notkestrasse 85, 22607 Hamburg, Germany \\ ${ }^{\S}$ Institut für Geowissenschaften, Facheinheit Mineralogie, Abt. Kristallographie, Universität Frankfurt, Altenhöferallee 1, 60438 \\ Frankfurt am Main, Germany \\ ${ }^{\perp}$ Mineralogisch-Petrographisches Institut, Universität Hamburg, Grindelallee 48, 20146 Hamburg, Germany \\ ${ }^{\mathrm{II}}$ European Synchrotron Radiation Facility, 38043 Grenoble, France
}

\section{Supporting Information}

\begin{abstract}
Time-resolved diffuse X-ray scattering is one powerful method for monitoring the progression from the creation of local structural changes inside a crystalline material up to the transformation of the whole crystalline bulk. In this work, we study the mechanism of phototransformation of a molecular crystal by time-resolved diffuse X-ray scattering. Here, an optical excitation source, like a pulsed laser, initiates structural transformations which are monitored by X-ray scattering techniques. We have studied the dimerization process of the molecular switch $\alpha$-styrylpyrylium (trifluoromethanesulfonate) TFMS, in particular for understanding whether cooperative effects influence the changes of the structure in the bulk and its periodicity. Upon illumination with optical light, $\alpha$-styrylpyrylium TFMS instantaneously photoswitches. Depending on the optical fluence, X-ray diffuse planes are observed prior to

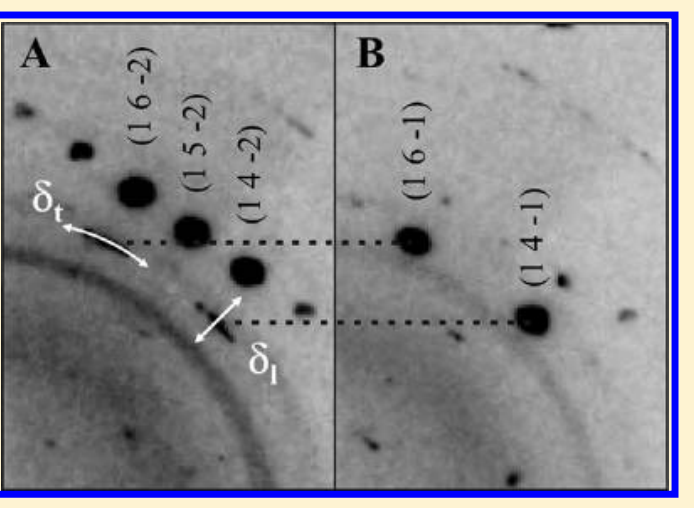
phototransformation of the bulk. In the early stages of transformation, the analysis reveals systems of randomly distributed islands of product clusters with gradual growth in size and amount. The degree of transformation follows the optical excitation profile, i.e., the spatial absorption of the laser beam. In the present studies, no influence of cooperativity on the photodimerization process has been found.
\end{abstract}

\section{INTRODUCTION}

Photoinduced processes in organic single crystals have been studied for a long time, and their potential in a variety of photosensitive devices has recently made them a focus of research. ${ }^{1-3}$ It has been shown that template-controlled reactions in organic solids can be used to construct functional organic building blocks with a wide range of applications like storage materials and macro- and microstructured reactors. ${ }^{4}$ For the creation of these frameworks, photoinduced dimerization might be one of the fundamental reactions which triggers the self-assembly. In the solid state, dimerization reactions proceed homogeneously or heterogeneously. ${ }^{5}$ In homogeneous reactions the crystal properties are conserved, whereas in heterogeneous reactions the periodicity of the crystal lattice is destroyed during the reaction. Though heterogeneous photodimerization reactions are widespread, homogeneous solid-state photoreactions are systematic studies on their reaction mechanism from a structural dynamics point of view rare. ${ }^{6}$ So far it is not clear how the optical photoexcitation conditions influence the topotacticity of the reaction (i.e., whether the reaction proceeds homogeneously or heterogeneously) and whether a low transformation degree follows the same mechanistic laws as the whole bulk transformation. Furthermore, it has not been investigated in detail to what extent ultrafast excitation conditions might lead to a different type of dimerization processes.

We believe that photocrystallography is the ideal tool for studying optical-induced reaction with structural changes in the solid state experiments ${ }^{7-12}$ and to investigate intermediates by time-resolved laser pump/X-ray probe experiments. ${ }^{13,14}$

In this work, we present our studies on phototriggered molecular switches, whose transformations are followed from local defined structures in the bulk up to the entire sample.

In this way, it becomes possible to give an overview on the transformation kinetics of solid-state reactions as a function of optical excitation fluence. Very low optical fluencies lead to a slow bulk transformation (optical illumination of the sample for hours) and allow quasi-static structural refinement with high

Received: March 2, 2012

Revised: August 22, 2012

Published: August 30, 2012 
spatial resolution at different grades of the transformation process. Using higher optical fluencies yielding in faster transformations (from $10 \%$ up to a complete transformation of the sample after a single optical pulse) of the bulk, investigations with high temporal resolution are possible. This study aims to understand to what extent the structural mechanistic properties are the same for all kind of excitation conditions and to compare these studies with ultrafast X-ray diffraction studies and their results. We have investigated $\alpha$ styrylpyrylium (trifluoromethanesulfonate) TFMS as an example, which bases on a chemical $[2+2]$ photodimerization reaction. ${ }^{16-18}$ Below $350 \mathrm{~K}$, the crystalline system undergoes an irreversible $[2+2]$ dimerization from the monomeric reactant state $\left(\mathrm{C}_{23} \mathrm{H}_{29} \mathrm{O}_{5} \mathrm{SF}_{3}\right)$ to the dimeric product state $\left(\mathrm{C}_{46} \mathrm{H}_{58} \mathrm{O}_{10} \mathrm{~S}_{2} \mathrm{~F}_{6}\right)$ by the absorption of visible light. At the absorption wavelength of $\lambda=444 \mathrm{~nm}$, this process reaches maximum efficiency. According to the Woodward-Hoffmann rules, ${ }^{19-21}$ the photodimerization is reversible above $350 \mathrm{~K}$ in this material: The absorption of light closes the ring system, thermal energy reopens it, and the crystal returns to the initial monomer state. During the structural switching, the periodic structure of $\alpha$-styrylpyrylium TFMS crystals remains intact in both directions of the reaction, thus allowing multiple switching cycles. In the following, ultrafast experiments are aiming for the time scales on the picosecond time scales and capitalize the reversible high-temperature state of the material. On the other hand, slow time scale experiments were carried out below 80 ${ }^{\circ} \mathrm{C}$ to investigate the bulk transformation from the monomer to the dimer state.

\section{EXPERIMENTAL SECTION}

Sample Preparation. $\alpha$-Styrylpyrylium TFMS was synthesized according to ref 22 . For the phototransformation experiments on slow time scales, $\alpha$-styrylpyrylium TFMS crystals with a size of about $200 \mu \mathrm{m} \times 150 \mu \mathrm{m} \times 150 \mu \mathrm{m}$ were used. For the ultrafast measurements, crystals with a maximum thickness of $500 \mathrm{~nm}$ and with edge lengths of $50 \mu \mathrm{m}$ $\times 100 \mu \mathrm{m}$ were used. The thickness of the crystals guarantees a homogeneous optical excitation and X-ray probing of the full volume. The crystals were grown on the surface of a metalcovered object holder by recrystallization of the material under slow evaporation of the solvent 1-hexanol.

Optical Spectroscopy. The phototransformation from the monomer to the dimer state was investigated by UV-NIR absorption spectroscopy (Cary-5E spectrometer, $0.2 \mathrm{~nm}$ bandpass). Further details concerning the spectroscopic setup can be found in ref 23. As samples, crystalline $\alpha$-styrylpyrylium TFMS pressed in $\mathrm{KBr}$ pellets was used. A closed-loop Oxford cooling system was used to perform the experiments at different temperatures above room temperature to induce the back reaction. Further information about the spectroscopic properties of the material can be found in ref 24 .

Photo X-ray Diffuse Scattering on Slow Time Scales. Photo X-ray diffuse scattering studies of $\alpha$-styrylpyrylium TFMS single crystals have been performed at beamlines F1 and D3 of the DORIS storage ring at HASYLAB at DESY. Details about the setups used can be found in refs 25 and 26 and in Figure 1. An X-ray energy of $20 \mathrm{keV}$ with an X-ray beam size of $0.4 \times 0.4 \mathrm{~mm}^{2}$ and a bandwidth of $\Delta E / E=5 \times 10^{-4}$ was used. The samples were mounted on a 4-circle diffractometer (D3) and on a Kappa diffractometer (F1). The crystals were measured with diffractometer angles $2 \theta=0^{\circ}, \omega=0^{\circ}, \chi=0^{\circ}$, and a $180^{\circ} \phi$-rotation in steps of $\Delta \phi=0.5^{\circ}$. The data

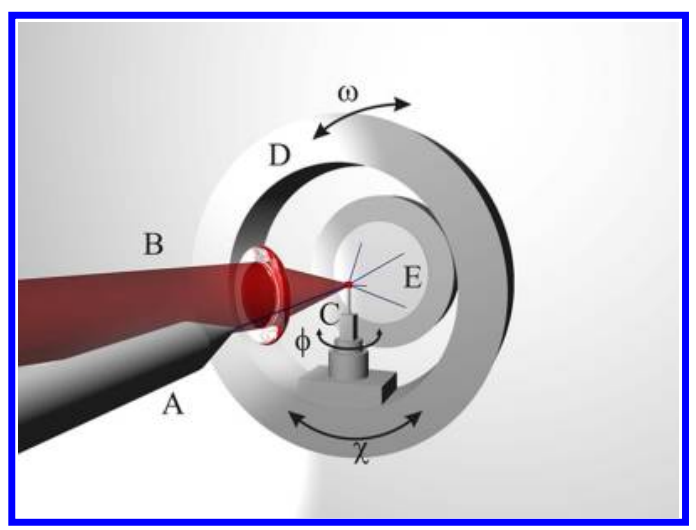

Figure 1. Three-dimensional graphic of the experimental setup used at the beamline D3, HASYLAB, Hamburg. The synchrotron beam (A) and the optical excitation light source (B) are almost collinear. The sample (C) is mounted on a 4-circle diffractometer (D) and the X-ray diffraction patterns are recorded using a MarCCD camera (E).

collection was done at $80 \mathrm{~K}$ using an open flow Oxford diffraction Helijet. A 2-dimensional detector (MarCCD 165) was used to record the scattering and diffraction patterns. The phototransformation was initiated by a cold-light source using an OG 580 optical edge filter, focused to $1 \mathrm{~mm}^{2}$ and providing a power of $20 \mathrm{~mW}$. The size of the sample and the photoexcitation parameter result in an optical density of the sample of $\mathrm{OD}_{580}=0.2$ for a crystal diameter of $200 \mu \mathrm{m}$, which ensures a homogeneous excitation. The molar fractions of monomer and dimer states were investigated as a function of the illumination time. Data evaluation, integration, and refinement were done using XDS. ${ }^{27}$ Crystallographic structure refinement has been done using the ShelXTL software. ${ }^{28}$

Picosecond Time-Resolved X-ray Scattering and Diffraction. The picosecond time-resolved measurements were performed on beamline ID09B at the ESRF. Further details concerning the general setup of the time-resolved experiment can be found in refs 29 and 30. In the ultrafast experiments, the photoreaction was initiated with $100 \mathrm{fs}$ optical pulses with a focal size on the sample of $1 \mathrm{~mm}^{2}$. The laser was synchronized to the synchrotron at a frequency of $986.3 \mathrm{~Hz}$, the 360th subharmonic of the orbit frequency in the synchrotron. The excitation wavelength of $520 \mathrm{~nm}$ was produced by a Ti:sapphire laser system with an OPG (optical parametric generator)/OPA (optical parametric amplification) unit providing a pump power of $25 \mu \mathrm{J}$. The wavelength setting, the illumination properties, and the sample size result in an optical density of $\mathrm{OD}_{520}=0.21$ for the laser beam in grazing incident on the sample. The monochromatic X-ray probe pulse (about 50 ps fwhm in average) had an energy of $16.5 \mathrm{keV}$ with a bandwidth of $\Delta E / E=3 \times 10^{-4}$. The X-ray focal size on the sample was $0.2 \mathrm{~mm}$ (horizontal) $\times 0.7 \mathrm{~mm}$ (vertical) on the sample. For the optical laser pump/X-ray probe excitation geometry, a quasi-parallel configuration was used. The sample geometry for X-ray diffraction was set to grazing incidence at an angle of $2^{\circ}$. For the search of the diffuse planes, the crystals were measured in rocking scan mode with a rocking range of $\Delta \phi=2^{\circ}$. A 2-dimensional detector (MarCCD 165) was used to record the $\mathrm{X}$-ray diffraction pattern.

\section{RESULTS AND DISCUSSION}

A priori it is not evident which growing law a photodimerization process follows (see Supporting Information). 
Experimentally, these mechanisms can be distinguished by the kinetic laws derived from the time evolution of the X-ray scattering and diffraction patterns. Under constant excitation conditions, it is also possible to distinguish between the mechanisms on the behavior of the $\mathrm{X}$-ray diffraction signal itself, i.e., the occurrence of diffuse X-ray scattering signals or the creation of Bragg reflections in reciprocal space. In the following, we will discuss both effects.

At low degrees of transformation, the X-ray scattering patterns vary differently in time, depending on the underlying propagation effect as explained before. The seed generation in the new dimer state leads to planes of diffuse scattering. These planes intersect the theoretical positions of the dimer Bragg peaks of a fully transformed crystal. During phototransformation, these diffuse planes grow in intensity and eventually take the shape of new Bragg diffraction peaks. If the occupancies of both monomer and dimer configuration are comparable, the Bragg reflections of both states are of appropriate intensities to extract structural information simultaneously for both states by standard crystallographic methods. We will summarize these results in the following and compare them to our optical investigations.

Growth Kinetics of Dimer Crystallites under Continuous Irradiation. In the following, we define dimer crystallites as domains with a dimer structure and an average sizes $>14 \mathrm{~nm}$. In order to determine the dimensionality of the phototransformation, the Johnson-Mehl-Avrami-Kolmogorow (JMAK) model ${ }^{31,32}$ was used to analyze the X-ray diffraction and the optical spectroscopy results, which was proved to be a strong tool ${ }^{3,33,34}$ and based on the time-dependent S-shaped function of the type $y=1-\exp \left(-k t^{n}\right)$, where $y$ is the dimer fraction formed during the irradiation time $t, k$ is the growth rate constant, and $n$ is the Avrami exponent.

The degree of transformation as a function of irradiation time is shown in Figure 2. Here, the results yielded from optical

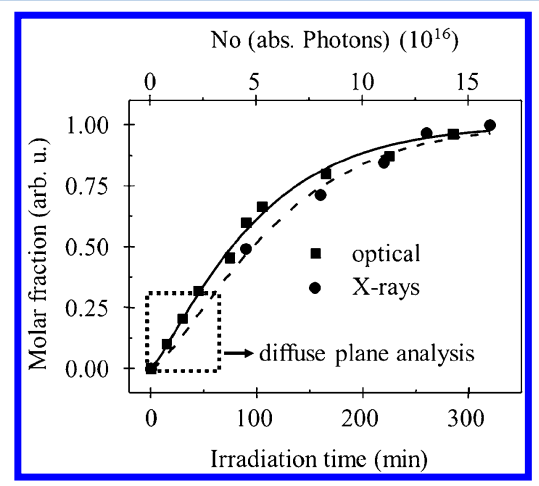

Figure 2. Molar fraction of dimers as a function of irradiation time determined from X-ray diffraction studies (circles) and the decrease of the integral optical absorption (squares) with its maximum at $444 \mathrm{~nm}$. The Avrami fits of the measurements lead to a dimensionality of 0.32 (X-ray diffraction measurement) and 0.13 (optical measurement), respectively. At low transformation degrees $(<30 \%)$ the $\mathrm{X}$-ray diffraction data can be treated by the diffuse plane analysis.

experiments are compared to the results based on photo X-ray crystallography studies. It probes the kinetics of a phase reaction including its nucleation and the dimensionality of the growth mechanism. The Avrami exponent of the fit is 1.32 leading to a dimensionality of the dimer state propagation of $\operatorname{dim}=0.32$. Optical investigations reveal a similar dimensionality of the phototransformation of $\alpha$-styrylpyrylium TFMS.
The Avrami exponent of the optical measurement was determined to 1.13 and the dimensionality of the propagation of the new state is 0.13 . The slow transformation of the bulk therefore suggests a 0 -dimensional transformation. The zerodimensionality of the reaction means that the dimer transformation probability does not depend on the kind of the neighboring molecules and surrounding lattice, but primarily on the absorption of optical photons. Therefore, cooperative effects based on phonons can be excluded.

The optical investigations of the back transformation to the monomer configuration show similar dimensionalities, but different rate constants depending on the temperature (Figure 3). The averaged Avrami exponent of the thermal back

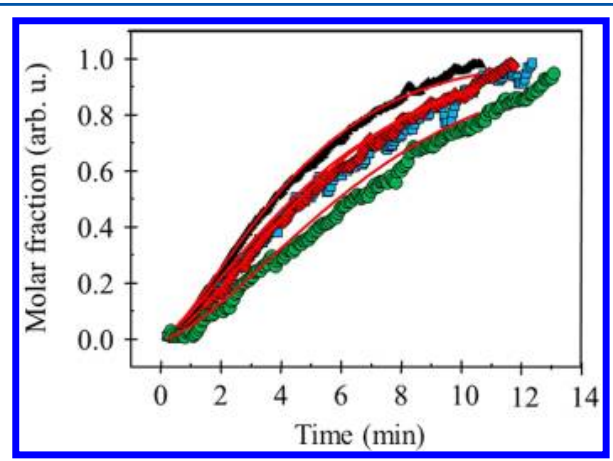

Figure 3. Change of molar fraction of monomers based on the temperature-induced back transformation to the monomer state recorded at $500 \mathrm{~K}$ (triangles, black), $470 \mathrm{~K}$ (rhombs, red), $430 \mathrm{~K}$ (squares, blue), and $400 \mathrm{~K}$ (circles, green). The percentage of the monomer phase measured by optical absorption measurements at different temperature can be fitted by an S-shape function (red lines) and results in an average dimensionality of the transformation of 0.4 .

reactions was determined from a set of absorption measurements at temperatures from 400 to $500 \mathrm{~K}$ to be $1.40 \pm 0.07$, leading to a dimensionality of 0.40 . It should be noted that no explicit temperature dependence of the Avrami parameter was found, so the dimensionality of the back transformation is statistical with respect to temperature.

As a result of these studies, more molecules are photodimerized in the illuminated front part, which explains the dimensionalities larger than 0 . The use of smaller samples or excitation with longer wavelengths would lead to a more homogeneous excitation and therefore to a smaller dimensionality, but, due to the worse signal-to-noise ratio upon weaker absorption of the photons in these cases, it is experimentally not suitable. Since optical spectroscopy normally monitors local changes and XRD techniques monitor the changes of the dimensionality of the bulk, the coincidence of the Avrami exponents of the phototransformation and its thermal back transformation emphasizes the statistical nature of both processes. In other words, we propose that forward and backward reactions are not driven by the crystal lattice.

Growth Kinetics of Dimer Seeds under Continuous Irradiation. In the following, we define the creation of dimer seeds as domains with a dimer structure and average sizes $<14$ $\mathrm{nm}$. Diffraction patterns of soft materials reflect the finite lattice coherence, as the longitudinal and transverse coherence length of the lattice planes. ${ }^{35}$ If no other phase-destroying effects apply, the longitudinal coherence length $\xi_{1}$ of the crystal is related to the finite crystallite size determined by grain boundaries. The transverse coherence length $\xi_{\mathrm{t}}$ of the crystal 
is related to the mean deviation angle of the lattice-plane normals with respect to the macroscopic orientation. The size of the crystal transverse coherence length $\xi_{\mathrm{t}}$ can also be limited by the finite transverse dimensions of the crystallites. As shown in the following, the critical parameters are met with dimer grain sizes of $\sim 14 \mathrm{~nm}$. We have used this size for our distinguishing between seed $(<14 \mathrm{~nm})$ and crystallite $(>14 \mathrm{~nm})$ formation.

Growth Kinetics of Seeds in the Longitudinal Direction. According to Scherrer ${ }^{35}$ for the special case of thin plate crystals, the minimum average crystallite size in the newly formed state can be determined by monitoring the full width at half-maximum (fwhm) of the reflections in longitudinal direction $\delta_{1}(2 \theta)$. For the definition of the longitudinal and transversal cuts through Bragg reflections, see Figure 4. With

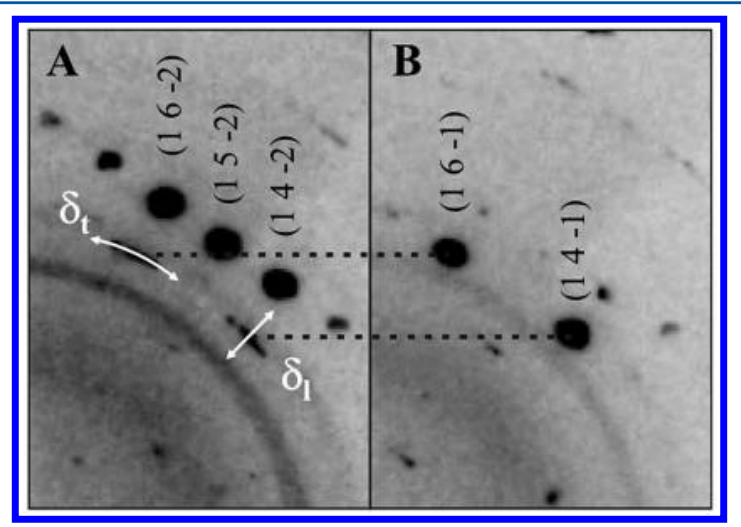

Figure 4. Bragg diffraction peaks of a crystal with a high monomer occupancy $(93 \%, A)$ and a low monomer occupancy (35\%, B). During the phase transformation, the diffuse planes change to Bragg peaks (1 $6-1)$ and $(14-1)$ of the dimer phase and the Bragg diffraction peaks $\left(\begin{array}{ll}1 & 6\end{array}-2\right),(15-2)$, and $(14-2)$ of the monomer phase disappear. The analysis of the diffuse $\mathrm{X}$-ray scattering is based on the fwhm in the directions $\xi_{\mathrm{t}}$ and $\xi_{1}$ for the transverse ( $\mathrm{t}$ ) and longitudinal (l) length, respectively. The circular X-ray scattering signal coinciding in both patterns is a result of ice formation during the measurement.

$\delta_{1}(2 \theta)=\lambda / l_{\text {ave }} \cos \theta_{0}$, it is possible to determine the average domain size $l_{\text {ave }}$ contributing to the diffraction signal. $\lambda$ is the Xray wavelength used and $\theta_{0}$ the diffraction angle at maximum intensity of the Bragg reflection. Note that in a fully evolved Bragg reflection, its fwhm is determined by the instrumental response function of the diffractometer. Therefore, Scherrer's equation sets a minimum limit of the observed crystallite sizes. In our case, this crystal size limit is $14 \mathrm{~nm}$ resembling the upper limit for the observable effect of growth kinetics of seeds.

Growth Kinetics of Seeds in the Transverse Direction. In order to understand the nucleation mechanism of the phototransformation, detailed analyses of the X-ray diffraction data at low transformation degrees (below 30\%) were performed with special emphasis on the appearance of diffuse planes upon light irradiation. In addition to the mechanism of domain formation, they also give indications on the size of the dimer domains formed. At transformation degrees around only $5 \%$, the full shape of the Bragg diffraction peaks is not developed and reflections with enlarged angular full width of half-maximum can be investigated. The analysis of the diffuse $\mathrm{X}$-ray scattering signals is based on the fwhm in the transverse (t) and longitudinal (l) directions $\delta_{\mathrm{t}}$ and $\delta_{\mathrm{l}}$, respectively (Figure 4).
The photoinduced diffuse X-ray signals are characterized by a transversal elongation rather than a longitudinal one. Therefore, and as the changes of the longitudinal coherence length in the crystal have already been discussed in the section before, we will now concentrate on the crystal transverse coherence length $\xi_{\mathrm{t}}$ and its changes upon photoillumination. As shown in Figure 4 , the Bragg diffraction peaks (1 $4-2),(15-2)$, and (1 $6-2)$ disappear during the phototransformation and the diffraction signals of the dimer $(14-1)$ and $(16-1)$ appear as weak diffuse lines. At two different time points during the transformation, the crystal used has dimer occupancies of $7 \%$ and $65 \%$ and the corresponding diffraction signals of the dimer changes from diffuse planes with a high angular fwhm (7\% dimer) to the common Bragg diffraction peaks $(14-1)$ and $(1$ $6-1)$ at a dimer occupancy of $65 \%$. The transverse crystal coherence length can be calculated by $\xi_{\mathrm{t}}=\delta_{\mathrm{t}} / \lambda$, where $\delta_{\mathrm{t}}$ is the observed fwhm of the diffuse scattering in transversal direction. Figure 5 shows the change of diffuse X-ray diffraction scattering

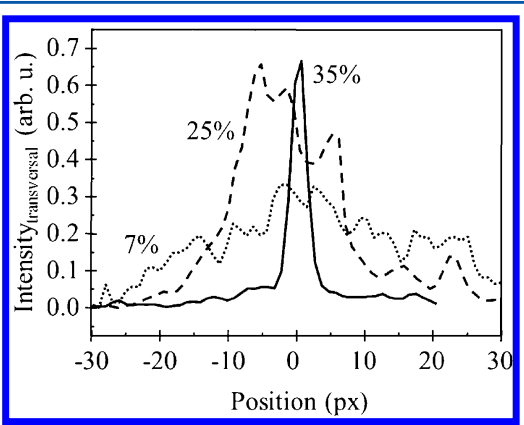

Figure 5. Change of the transversal fwhm of diffuse X-ray diffraction scattering to Bragg diffraction peaks upon light irradiation (as transversal intensity changes in arbitrary units versus pixel position on the detector). Here, the fwhm at dimer occupancies of $7 \%, 25 \%$, and $35 \%$ are shown which decrease upon the phototransformation.

to a Bragg diffraction peak upon light irradiation (as transversal intensity changes in arbitrary units versus pixel position on the detector). As an example, the fwhm at dimer occupancies of $7 \%, 25 \%$, and $35 \%$ are shown, which decrease upon the phototransformation. In the case of the dimer diffraction signal ( $14-1)$, the spatial intensity distribution evidently narrows and the fwhm decreases at larger dimer occupancies.

Figure 6 shows the transverse coherence length at different transformation degrees, based on the diffraction signal of reflections $(71-1),(6-3-2),(5-3-1)$, and $(4-2-10)$, which grow from the diffuse planes toward normal Bragg diffraction peaks (see also Figure 4). The average size of the dimer sections of the crystal increases linearly with the percentage of dimer occupancy. At 35\% dimer population, the transverse coherence length reaches a plateau and the Bragg peaks belonging to the dimer are completely developed. Again, at transformation degrees above $35 \%$, due to the limits of the apparatus response function, the dynamics of the nucleation cannot further be monitored. We have assigned this limit as the dashed line in Figure 6. Using the JMAK model to analyze the time dependence of the fwhm of the Bragg diffraction peak and the diffuse planes, the fit leads to a dimensionality of 0.83 . However, this value is a convolution of instrumental response function and JMAK kinetic properties of the sample. The instrumental response function has been determined by investigating the change of the fwhm at high transformation degrees. Combining the X-ray diffuse scattering with the 


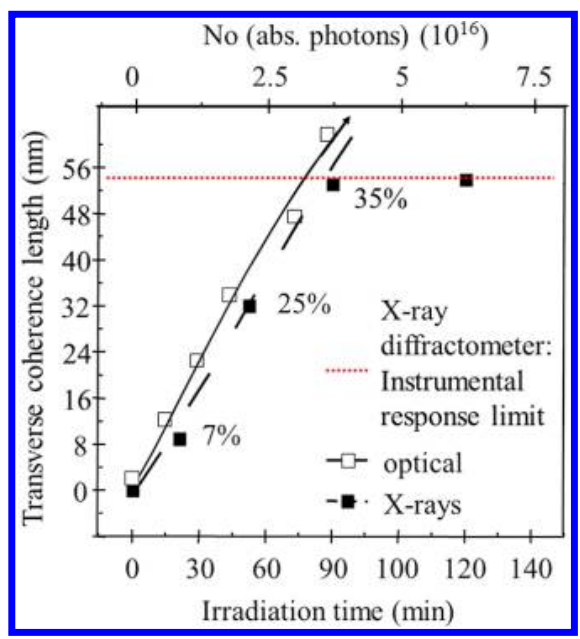

Figure 6. Transverse coherence lengths in an $\alpha$-styrylpyrylium TFMS crystal against the irradiation time. The dimer state has been populated by light-induced dimerization at $580 \mathrm{~nm}$ with a power of $20 \mathrm{~mW}$. At occupancies of around $30 \%$ and a corresponding transverse coherence length of about $45 \mathrm{~nm}$, the dimer diffraction peaks can be identified as Bragg peaks and the instrumental function is dominant. The data were determined, e.g., by the observed reflections $(71-1),(6-3-2)$, (5 $-3-1)$, and $(4-2-10)$. The Avrami fits based on the optical measurements (solid line) and the X-ray diffraction data (dashed line) showing the conformance to the diffuse X-ray scattering results.

diffraction work, i.e., kinetic investigations of seed growth below and above $14 \mathrm{~nm}$ within the same data set, gives an overall dimensionality of 0.24 . Hereby, the coherence lengths of the kinetic investigation data were calculated, using the values $0 \%$ and $35 \%$ as reference points. This dimensionality agrees well with the optical measurements and clearly proves the expected mechanism in which no cooperativity or phonon propagation is needed for photoswitching-only pure absorption of optical photons.

In summary, the current analysis reveals the creation of randomly distributed islands of product domains gradually growing in size as the reaction proceeds. The degree of transformation follows the optical excitation profile, i.e., the spatial absorption of the illuminating light. The slow kinetics diffraction experiments reveal the simple dependence of the integral flux in the excitation pulse on the instantaneously created seeds with random distribution.

Simulation of X-ray Diffuse Scattering Signals in Crystalline $\boldsymbol{\alpha}$-Styrylpyrylium TFMS. Figure 7 summarizes the results for the simulation of the X-ray diffuse scattering signal changes for various phototransformation mechanisms in crystalline $\alpha$-styrypyrylium TFMS. The simulations were performed using the software package Discus. ${ }^{36}$ For the simulations, a crystal size of about $140 \mathrm{~nm}$ diameter was chosen, corresponding to edge lengths of $100 \times 100$ unit cells. The simulations were started from a pure monomer crystal and ended in the simulations of a pure dimer crystal. Changes of Xray scattering signals of various transformation rates were calculated and are shown for $10 \%$ of phototransformation in Figure 7. In order to simulate the differences between a 0dimensional transformation mechanism and a 1-dimensional transformation mechanism for an overall phototransformation of $10 \%$, the size of the randomly distributed dimer seeds has additionally been varied.

The simulation of the diffraction for the pure monomer crystal (Figure 7A) and pure dimer crystal (Figure 7E)

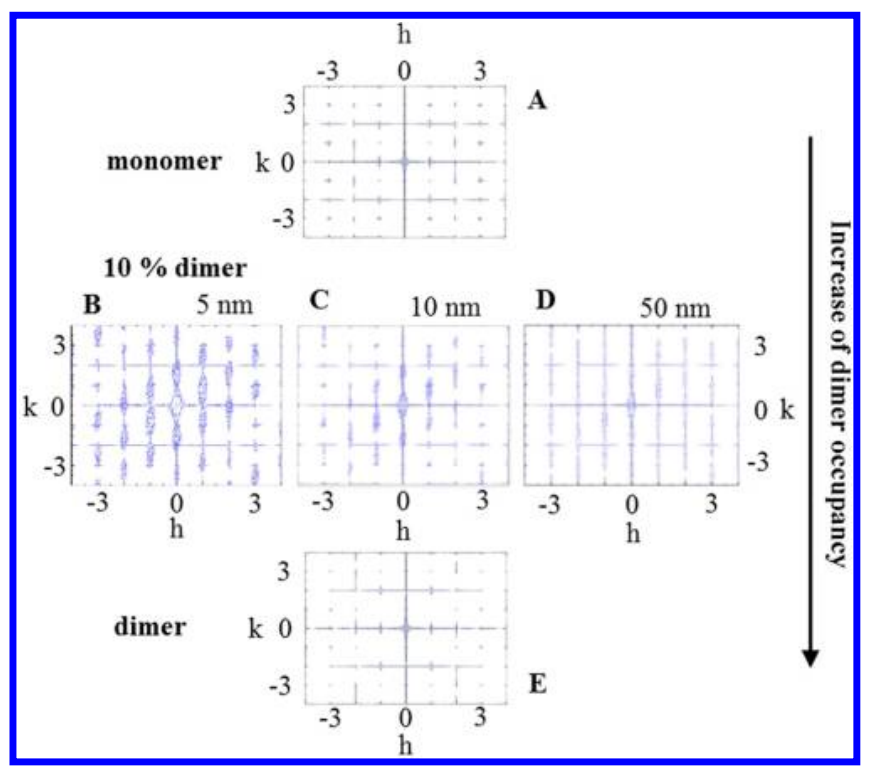

Figure 7. Simulated changes of X-ray scattering behavior of $\alpha$ styrylpyrylium TFMS crystal during photodimerization. (A) Diffraction of pure monomer crystal. (B-D) Scattering properties of $10 \%$ of photodimerized crystal: (B) diffuse X-ray scattering caused by the creation of $5 \mathrm{~nm}$ dimer seeds; (C) diffuse X-ray scattering caused by the creation of $10 \mathrm{~nm}$ dimer seeds; (D) Bragg diffraction caused by the creation of $50 \mathrm{~nm}$ big dimer seeds. (E) Diffraction of pure dimer crystal.

essentially reveal the expected reciprocal space patterns as expected according to their unit cell dimensions and crystal symmetry. The simulations of these phases have been performed for the Miller indices $h=(-4)$ to $h=4, k=$ $(-4)$ to $k=4$, and $l=0$.

For the simulations of the X-ray diffraction pattern at $10 \%$ phototransformation (Figure $7 \mathrm{~B}-\mathrm{D}$ ), the same range of Miller indices has been used. Characteristic for the 0-dimensional transformation mechanism is the formation of a number of little seeds directly upon photoexcitation. In the simulation, these seeds are presented by randomly oriented dimer domains of the size of about $5 \mathrm{~nm}$ in diameter (corresponding to about $3 \times 3$ dimer unit cells). The creation of these seeds leads to a pure diffuse X-ray scattering signal and confirm the experimental results summarized in Figure 6. In Figure 6, 7\% of phototransformation leads to the creation of dimer seeds with an average size of $8 \mathrm{~nm}$ (simulated: $5 \mathrm{~nm}$ seeds at $10 \%$ transformation) which coincides within the error bar of the simulations and the experiment. Simulating 2 times larger dimer seeds (10 nm diameter) randomly distributed within a $140 \mathrm{~nm}$ sized monomer crystal again leads to the creation of diffuse planes (Figure 7C).

For a 1-dimensional transformation, directly after photoexcitation the seed sizes are considerably larger due to cooperativity effects. Cooperativity means that in a cascade reaction one photon initiates more than one reaction to a dimer. ${ }^{37}$ Simulations base on a very conservative value of 10:1 as the ratio for the number of excited molecules to the number of absorbed optical photons. We have simulated that effect by choosing 10 times larger dimer seeds of $50 \mathrm{~nm}$, again randomly distributed in the $140 \mathrm{~nm}$ sized monomer crystal. This kind of dimer seeds lead to well-defined Bragg diffraction peaks, as shown in Figure 7D. Projecting that result in Figure 6, it would mean that Bragg diffraction peaks have already been formed 
and the diffuse X-ray scattering signal have been disappeared upon an optical photon flux of about $10^{16}$ absorbed photonsessentially meaning a quasi-linear dependency of the transverse coherence length with increasing optical photon flux with a gradient a factor 10 steeper as the one experimentally observed in Figure 6.

The simulations of Figure 7 in conjunction with the experimental results of Figure 6 emphasize that for a given Xray flux the creation of diffuse X-ray scattering planes or welldefined Bragg peaks can be used to distinguish between a 0dimensional and 1-dimensional transformation mechanism (for further information see Supporting Information).

Ultrafast Creation of Dimer Crystallites. In the following, we will finish our discussion by reflecting the timedependent behavior of the ultrafast switching of $\alpha$-styrypyrylium TFMS bulk investigated with ultrafast X-ray diffraction. For a valid comparison of the experiments, the same level of optical absorbed photons and at the same transformation rates need to be considered. Then it is possible to use Figures 6 and 7 as references distinguishing whether the phototransformation mechanism investigated with ultrafast X-ray diffraction differs from the ones observed under slow quasi-continuous wave excitation conditions.

Effectively, the conversion rate of the ultrafast diffraction experiment was determined to be $20 \% \pm 8 \%$. Figure 8 A shows the radial intensity profile of the Bragg diffraction peak ( $\left.\begin{array}{lll}2 & 2 & 1\end{array}\right)$ at different time points measured at the time-resolved beamline ID09B, ESRF. As shown, at early (-100 ps) and late (100 ps) time points, only the Bragg diffraction peak (2 211$)$ of the monomer phase is observable. Around time zero, however, when the X-ray pulse and laser pulse are temporally overlapped,

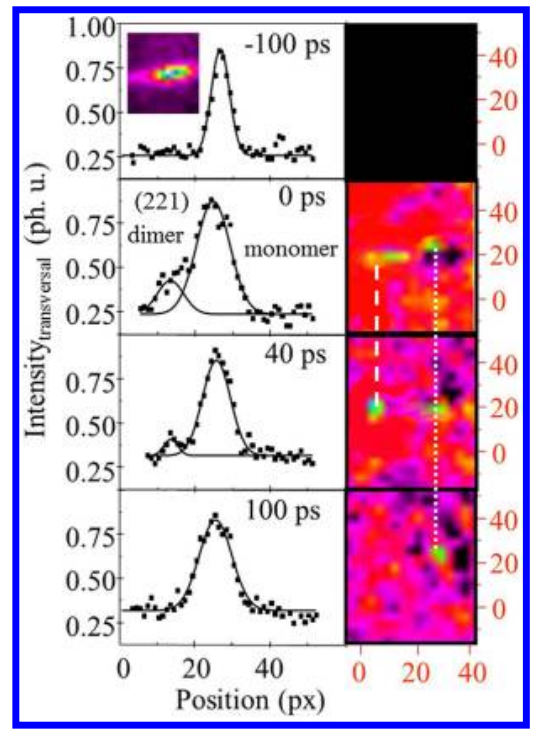

Figure 8. (A, left) Radial intensity profile of the Bragg diffraction peak ( $\left.\begin{array}{lll}2 & 2 & 1\end{array}\right)$ at different time points measured at the time-resolving beamline ID09B, ESRF. At $-100 \mathrm{ps}$ and $+100 \mathrm{ps}$, the Bragg diffraction peak of the monomer phase can be seen, whereas at 0 and 40 ps an additional contribution of the dimer phase to the Bragg diffraction peak can be identified with dimer domain formation larger than 14 $\mathrm{nm}$. Due to a temperature-induced back transformation into the monomer state, the dimer contribution disappears until the time point +100 ps. (B, right) Intensity difference map of the collected Bragg reflection positive time points minus negative time point $(-100 \mathrm{ps})$ emphasizing the ultrafast formation of a whole domain. Note that the difference map is evidently empty at $t=-100 \mathrm{ps}$. a satellite peak ( 0 and $40 \mathrm{ps}$ ) is observed next to the Bragg peak which is associated with the dimer formation $((221)$ of the dimer phase). As described in ref 16, the ring formation and ring-opening reactions of $\alpha$-styrypyrylium TFMS are very fast and within the time resolution of the experimental setup.

Figure $8 \mathrm{~B}$ summarizes the intensity difference map of the collected Bragg reflection positive time points minus negative time point $(-100 \mathrm{ps})$ emphasizing the ultrafast formation of the whole domain. Note that the difference map is evidently empty at $t=-100 \mathrm{ps}$. Disappearance of the dimer contribution at +100 ps was initiated using a high experiment temperature $\left(+100{ }^{\circ} \mathrm{C}\right)$. In this way, the back transformation was directly released and the sample returned into the monomer state.

For the ultrafast diffraction experiment, the shape analysis of the instantaneously formed Bragg diffraction peak reveals the formation of dimer seeds larger than $14 \mathrm{~nm}$. This result coincides nicely with the measured transversal coherence lengths in Figure 6 and the prediction/simulation given in Figure 7D. At those transformation rates, including the error bar of the determination of the conversion rates, dimer seed sizes between 15 and $20 \mathrm{~nm}$ are predicted. According to Figures 6 and 7, a decrease of the optical photon flux by a factor of 10 , corresponding to a conservative approach of cooperativity mechanism or a 1-dimensional phototransformation mechanism, would mean that the Bragg diffraction peak remain nearly constant if laser flux is lowered. However, experimentally, the Bragg diffraction peak of the dimer state is not measurable anymore when the optical photon flux is lowered by a factor of 5 .

Including the results of the time-dependent analysis given by the application of the JMAK model, we can safely conclude that in the case of $\alpha$-styrylpyrylium TFMS independent of the excitation conditions like optical flux, transformation degree, or the use of quasi-continuous excitation or excitation with ultrafast X-ray pulses, the photodimerization in the crystalline phase follows a 0-dimensional growing model. Under the optical excitation conditions used, the dimer formation is purely photon-driven and does not need cooperative lattice support. In Figure 9, the time-dependent behavior of the integral

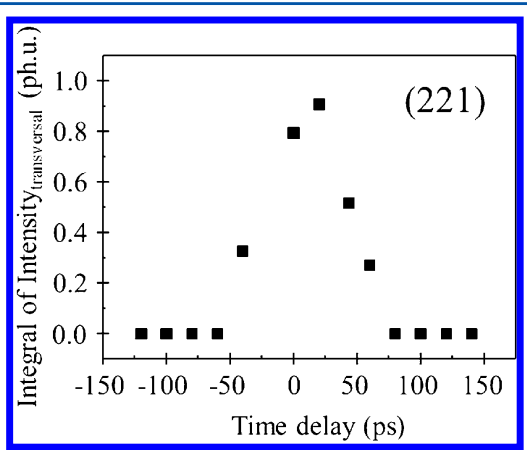

Figure 9. Time evolution of the integral intensity of the (2 $\left.2 \begin{array}{ll}2 & 1\end{array}\right)$ dimer satellite peak at ultrafast time scales. The temporal fit is Gaussian shaped and 53 ps wide, resembling the time-resolution of the synchrotron experimental setup.

intensities of this light-induced contribution to the Bragg diffraction peak ( $\left.\begin{array}{lll}2 & 2 & 1\end{array}\right)$ is described as Gaussian-shaped. The temporal fwhm has found to be 53 ps which very much resembles the time resolution of the apparatus. In the present experiment, the 0 -dimensional transformation mechanism is therefore nearly instant and forms coherent dimer domains/ 
seeds larger than $14 \mathrm{~nm}$. The transformation mechanism does not depend on coherence or wavelength of the absorbed optical light nor the flux of the absorbed photons but is only a material specific property. Furthermore, the system shows neither an effect of laser heating on the dimerization process nor an effect of higher process temperatures of the back transformation (up to $160{ }^{\circ} \mathrm{C}$ ).

\section{CONCLUSION}

In summary, the photodimerization of $\alpha$-styrylpyrylium TFMS has no propagation direction during the photoreaction which is phonon-supported. The Avrami fits of the X-ray diffraction data for grains smaller and grains larger than $14 \mathrm{~nm}$, as well as for the optical absorption experiments, indicate very much similar kinetic behavior. The growth of the dimer nucleation points within the crystal and the grain growth of seeds smaller than 14 $\mathrm{nm}$ have been investigated by the analysis of diffuse planes in the X-ray diffraction data. The growth kinetics of larger seeds has been characterized by crystallographic techniques. By investigating the fwhm of the diffuse reflections, the average sizes at different transformation degrees were analyzed up to the formation of the pure dimer state. Analysis of the coherence length, using the JMAK model at low transformation degrees, shows good agreement with the X-ray diffraction data at high degrees of transformation. The diffuse scattering experiments also reveal further important aspects on the mechanism of phototransformation: (a) during the dimerization by growing from the local seeds to the global crystal in total, the change of the angle of the unit cell dimensions must occur in a coherent way, otherwise, the crystal would not transform homogeneously; (b) as the finite transverse dimensions of the crystallites of the photodimerized crystallites grow-from the local seeds to the overall global crystal-the photoinduced changes of the unit cell parameters during dimerization lead to a disorder effect in the crystal. They are reflected in changes of the mean deviation angle of the lattice-plane normals with respect to the macroscopic orientation. This can be observed as an increase of the transverse crystal coherence length.

The optical excitation conditions obviously lead to a uniform transformation mechanism independent of the seed size. No cooperative or phonon-supported mechanism has been found for the dimerization reaction under study. On the ultrafast time scale, no diffuse X-ray scattering has been observed. High optical photon flux excitation results in an instantaneous and homogeneous formation of the dimer state with domain formations larger than $14 \mathrm{~nm}$ in seed size. The photodimerization in the crystalline phase of $\alpha$-styrylpyrylium TFMS follows a 0-dimensional growing model. Kinetic analysis and reciprocal space analysis (diffuse $\mathrm{X}$-ray scattering) reveals a dimerization process independent of the excitation conditions like optical flux, transformation degree, or the use of quasicontinuous excitation or excitation with ultrafast X-ray pulses.

\section{ASSOCIATED CONTENT}

\section{S Supporting Information}

Description of the different growing laws of photodimerization processes. This material is available free of charge via the Internet at http://pubs.acs.org.

\section{AUTHOR INFORMATION}

\section{Corresponding Author}

*E-mail: stecher@gwdg.de.

\section{Notes}

The authors declare no competing financial interest.

\section{ACKNOWLEDGMENTS}

This work was supported by SFB 602 of the Deutsche Forschungsgemeinschaft and NEST-MI-3567. S.T. is grateful to the DFG (TE347, 1-3), Aventis Foundation and Fonds of the Chemical Industry. S.T. and J.H. thank the Advanced Study Group of the Max Planck Society for continuous support. R.M. thanks the SFB 602.

\section{REFERENCES}

(1) Garcia-Garibay, M. A. Angew. Chem., Int. Ed. 2007, 46, 89458947.

(2) Kuzmanich, G.; Xue, J.; Netto-Ferreira, J.-C.; Scaiano, J.-C.; Platz, M.; Garcia-Garibay, M. A. Chem. Sci. 2011, 2, 1497-1501.

(3) Cao, D.-K.; Sreevidya, T. V.; Botoshansky, M.; Golden, G.; Benedict, J. B.; Kaftory, M. J. Phys. Chem A 2010, 114, 7377-7381.

(4) Hamilton, T. D.; Papaefstathiou, G. S.; MacGillivray, L. R. J. Solid State Chem. 2005, 178, 2409-2413.

(5) Busse, G.; Tschentscher, Th.; Plech, A.; Wulff, M.; Frederichs, B.; Techert, S. Faraday Discuss. 2003, 122, 105-117.

(6) Theocharis, C. R.; Desiraju, G. R.; Jones, W. J. Am. Chem. Soc. 1984, 106, 3606-3609.

(7) Coppens, P. Synchrotron Radiat. News 1997, 10 (1), 26-30.

(8) Kim, C. D.; Pillet, S.; Wu, G.; Fullagar, W. K.; Coppens, P. Acta Crystallogr. 2002, A58, 133-137.

(9) Allen, F. H.; Mahon, M. F.; Raithby, P. R.; Shields, G. P.; Sparkes, H. A. New J. Chem. 2005, 29 (1), 182-187.

(10) Techert, S.; Zachariasse, K. A. J. Am. Chem. Soc. 2004, 126, $5593-5600$

(11) Collet, E.; Lemée-Cailleau, M.-H.; Buron-Le Cointe, M.; Cailleau, H.; Wulff, M.; Luty, T.; Koshihara, S.-Y.; Meyer, M.; Toupet, L.; Rabiller, P.; et al. Science 2003, 200, 612-615.

(12) Collet, E.; Buron-Le Cointe, M.; Cailleau, H. J. Phys. Soc. Jpn. 2006, 75, 11002-1-9.

(13) Techert, S.; Schotte, F.; Wulff, M. Phys. Rev. Lett. 2001, 86, 2030-2033.

(14) Rajkovic, I.; Busse, G.; Hallmann, J.; More, R; Petri, M; Quevedo, W.; Krasniqi, F.; Rudenko, A.; Tschentscher, T.; Stojanovic, N. S.; et al. Phys. Rev. Lett. 2010, 104, 125503-125506.

(15) Rajkovic, I.; Hallmann, J.; Grübel, S.; More, R.; Quevedo, W; Petri, M.; Techert, S. Rev. Sci. Instrum. 2010, 81, 045105-1-6.

(16) Hallmann, J.; Morgenroth, W.; Paulmann, C.; Davaasambuu, J.; Kong, K.; Wulff, M.; Techert, S. J. Am. Chem. Soc. 2009, 131, 1501815025 .

(17) Novak, K.; Enkelmann, V.; Wegner, G.; Wagener, K. B. Angew. Chem., Int. Ed. Engl. 1993, 32, 1614-1616.

(18) Enkelmann, V.; Wegner, G. J. Am. Chem. Soc. 1993, 115, 10390-10391.

(19) Zimmermann, H. E.; Nesterov, E. E. Acc. Chem. Res. 2002, 35, $77-85$.

(20) Woodward, R. B.; Hoffmann, R. J. Am. Chem. Soc. 1965, 87, 395-397.

(21) Keating, A. E.; Garcia-Garibay, M. A. Photochemical Solid-ToSolid Reactions. In Organic and Inorganic Photochemistry; Ramamurthy, V., Schanze, K., Eds.; Marcel Dekker: New York, 1998; Vol. 2, pp $195-248$.

(22) Hesse, K.; Hünig, S. Liebigs Ann. Chem. 1985, 105, 715-739.

(23) Techert, S.; Wiessner, A.; Schmatz, S.; Staerk, H. J. Phys. Chem. 2001, B105, 7579-7587.

(24) Hallmann, J.; Techert, S. J. Phys. Chem. Lett. 2010, 1, 959-961.

(25) Grabowsky, S.; Pfeuffer, T.; Morgenroth, W.; Paulmann, C.; Schirmeister, T.; Luger, P. Org. Biomol. Chem. 2008, 6, 2295-2307.

(26) Morgenroth, W.; Overgaard, J.; Clausen, H. F.; Svendsen, H.; Jørgensen, M. R. V.; Larsen, F. K.; Iversen, B. B. J. Appl. Crystallogr. 2008, 41, 846-853.

(27) Kabsch, W. J. Appl. Cryst. 1993, 26, 795-800. 
(28) Sheldrick, G. M. Acta Crystallogr. 2008, A64, 112-122.

(29) Schotte, F.; Techert, S.; Anfinrud, P.; Srajer, V.; Moffat, K.; Wulff, M. Recent Advantages in the Generation of Pulsed Synchrotron Radiation Suitable for Picosecond Time-resolved X-Ray Studies. In

Third-Generation Hard X-ray Synchrotron Radiation Sources; Mills, D. M., Ed.; Wiley-Interscience: New York, 2001; pp 345-369.

(30) Davaasambuu, J.; Durand, P.; Techert, S. J. Synchrotron Radiat. 2004, 11, 483-489.

(31) Avrami, M. J. Chem. Phys. 1939, 7, 1103-1112.

(32) Avrami, M. J. Chem. Phys. 1941, 9, 177-184.

(33) Benedict, J.; Coppens, P. J. Phys. Chem. A 2006, 110, 32613265.

(34) Bertmer, M.; Nieuwendaal, R. C.; Barnes, A. B.; Hayes, S. E. J. Phys. Chem. B 2006, 110, 6270-6273.

(35) Guinier, A. X-Ray Diffraction: In Crystals, Imperfect Crystals, and Amorphous Bodies; Dover Publications Inc.: Mineola, NY, 1994.

(36) Neder, R. B.; Proffen, T. Diffuse Scattering and Defect Structure Simulations; Oxford University Press: Oxford, UK, 2008.

(37) Koshino, K.; Ogawa, T. J. Phys. Soc. Jpn. 1998, 67, 2174-2177. 